\title{
DEVELOPMENT OF A NITRIDATION GAS-SURFACE BOUNDARY CONDITION FOR HIGH-FIDELITY HYPERSONIC SIMULATIONS
}

\author{
Michele Capriati ${ }^{1}$, Krishna S. Prata ${ }^{2}$, Thomas E. Schwartzentruber ${ }^{2}$, Graham V. \\ Candler $^{2}$, Thierry E. Magin ${ }^{1}$ \\ ${ }^{1}$ von Karman Institute, Waterloosesteenweg 72, 1640 Sint-Genesius-Rode (BE), \\ michele.capriati@vki.ac.be, magin@vki.ac.be \\ ${ }^{2}$ University of Minnesota, 55455 Minneapolis, Minnesota (USA), prata010@umn.edu, \\ schwart@umn.edu, candler@umn.edu
}

Key words: Gas-Surface Interaction, Atmospheric Entry Flows, Hypersonics, Computational Fluid Dynamics

\begin{abstract}
Gas-surface interaction phenomena have a strong impact on the heat flux experienced by atmospheric entry bodies in the hypersonic regime. Numerically, they can be expressed as a boundary condition to be imposed to the Navier-Stokes equations to achieve predictive engineering simulations. The mass and energy conservation can be abstracted in a thin layer containing both the solid and the gas phases. Such a balance was implemented in the open source MUTATION++ library. It is convenient to easily plug verified models in any type of CFD solver to model the response of material surfaces. We have extended the library to accommodate a state-of-the-art nitridation and nitrogen recombination mechanisms derived from beam experiments. MUTATION++ was coupled to US3D, a high-fidelity finite-volume flow solver, to simulate an experimental campaign conducted in the VKI Plasmatron facility. The experiment consists in applying a subsonic high-enthalpy nitrogen flow over an axi-symmetric ablative material sample. The simulation results on the stagnation line were compared to those obtained using a one-dimensional solver. Both results showed good agreement, verifying the implementation of the boundary condition. The computational model predicts a lower mass blowing rate than the experimental value. The catalytic behaviour of the mechanism, in agreement with the beam experiment predictions, induces higher heat flux values than those expected for the testing conditions of the Plasmatron facility.
\end{abstract}

\section{INTRODUCTION}

The development of predictive engineering numerical tools combines three ingredients: physico-chemical models, computational methods, and experiments. Transport processes in hypersonic flows can be described by means of partial differential equations that express the conservation of mass, momentum and energy in the system. These equations are generally solved choosing an appropriate discretization method on a numerical grid representing the physical domain of interest. In addition to the numerical accuracy of the computational method, the reliability of engineering predictions strongly depends on the fidelity of the physico-chemical models used to close the governing equations. Hypersonic flows span a broad range of temporal scales, from local thermodynamic equilibrium to thermo-chemical nonequi- 
librium states. Equations are implemented in a flow solver that provides the framework for numerical simulations. The implementation needs to be verified for assessing how close it is to a representation of the mathematical formulation. Then, the computational model needs to be validated against experiments, for assessing how correctly the model predicts a phenomenon. In this article, we focus on Gas-Surface Interactions (GSIs).

Accurately modeling GSI phenomena is crucial to predict the heat flux experienced by atmospheric entry bodies in the hypersonic regime. As particles cross the bow shock, their thermal energy drastically increases, leading to excitation of the internal energy modes, as well as dissociation and ionization of the gas: a chemically reacting boundary layer develops in front of the vehicle. GSIs describe the set of phenomena occurring between the reactive gas and the material surface, involving heterogeneous chemical reactions together with mass and heat exchange. It is a complex/multidisciplinary problem. CFD simulations are essential to retrieve the state of the gas interacting with the surface. This interaction can be predicted by (weakly or strongly) coupling the CFD solver to a material response code, which makes the simulations very computationally expensive. Another strategy is to model this interaction as a boundary condition, the latter approach is followed in this study.

In previous works, GSI models were abstracted in the MUTATION++ open source software $[1,2]$ to provide a BC for chemical reacting surfaces. The MUlticomponent Thermodynamic And Transport properties for IONized gases library written in $\mathrm{C}++($ MUTATION++) has been developed at the von Karman Institute to help centralize physico-chemical models, algorithms, and data into a single software package, which may be shared among several CFD tools. This presents several advantages: I) the equations implemented in the library can be verified separately, and the CFD code verification process
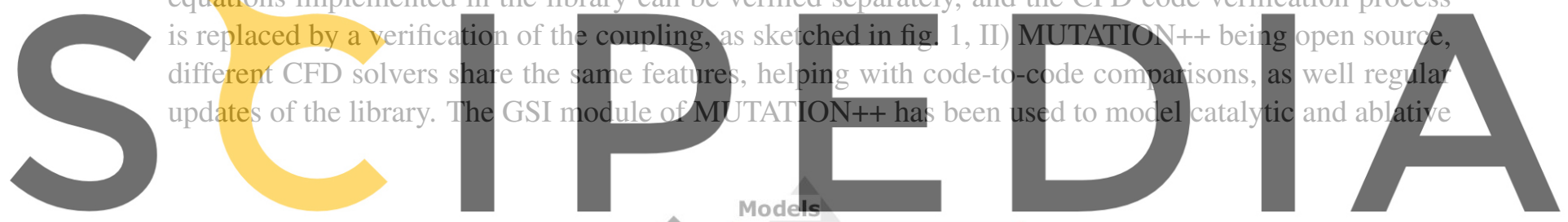

Register for free at https//www.scipedia.com to download the version without the watermark

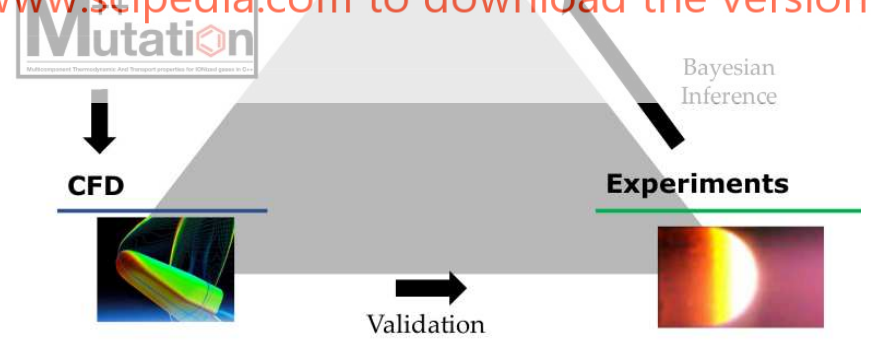

Figure 1: Developing predictive engineering tools for hypersonic flow applications: CFD is a key link between models and experiments.

materials, both assuming thermal equilibrium and non-equilibrium (state-to-state catalysis or two temperature ablation) of the gas near the surface $[1,3]$.

In this work, we extend the library to use a finite-rate chemistry model derived from beam experiments [4], for describing, among other reactions, surface nitridation and nitrogen recombination [5]. 
Such a state-of-the-art model provides a description of the chemical and fluid aspects of ablation, including pressure dependence and surface coverage, aiming at better predictions of the surface properties (heat flux, recession rate, etc.). The work is divided in two parts:

- Validate the model using the VKI stagnation line flow solver [6] coupled to MUTATION++ to study nitridation in an experimental campaign for a graphite material in a low-pressure hightemperature nitrogen plasma flow obtained in the Plasmatron facility of the von Karman Institute [7].

- Couple the MUTATION++ library to a high-fidelity solver, US3D [8], with the aim to account for more complex geometries. The coupling was verified by simulating the same experimental campaign using US3D and comparing the results obtained along the stagnation line with the results provided by the VKI stagnation line solver.

The paper is structured as follows: in sec. 2) the modeling of a chemically reacting surface is presented, including the definition of the chemical production terms; in sec. 3) the governing equations and the CFD solvers are described; in sec. 4) the experimental campaign is briefly illustrated; in sec. 5) the validation of the model is assessed through numerical simulations using the VKI stagnation line solver; followed by the verification of the MUTATION++ coupling with the US3D solver; lastly, the conclusions and future work are reviewed in sec. 6).

\section{GAS-SURFACE INTERACTION}

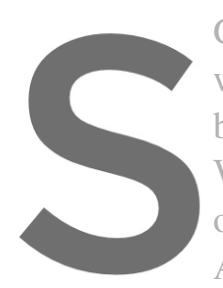

GSI phenomena can be

when dealing with a chem cally

by solving balance equati

When the surface temper

of the species partial densities.

Assuming the surface recession
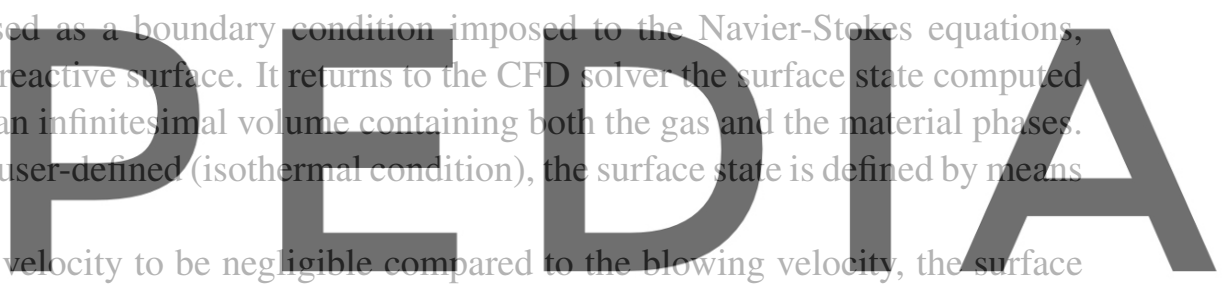

mass balance for each species i reads:

Register for free at https//www.scipediá.€(pno tojdownloads,the version without the watermark

where symbol $S$ stands for the set of species indices and $\mathrm{n}$ is the normal to the surface. The term

$\mathbf{u}_{\mathrm{g}}=\sum_{i \in S} \omega_{\mathrm{i}}^{\mathrm{s}} / \rho$ is the biowing velocity of the gas at the interface as a result of abiation processes, $\rho_{\mathrm{i}}$, the mass density of species $i, \rho$ the mass density of the gas mixture, and $\mathbf{j}_{\mathrm{i}}$ the diffusion mass flux of species $i$. The term $\dot{\omega}_{\mathrm{i}}^{\mathrm{s}}$ is the chemical production rate of species i. It can be computed using phenomenological or finite-rate chemistry models, as described in the following sections. Different fidelity models for chemical closures are available in MUTATION++ [1]. The gas state near the interface is obtained by solving the system (1) using a Newton method and the surface state is returned to the CFD solver.

When the temperature at the wall is unknown, an additional energy balance must be applied. Such a balance is also available in MUTATION++, but was not used in this work.

\subsection{Phenomenological approach}

Phenomenological models were first introduced to describe the catalytic behaviour of a surface in hypersonic simulations [9], and later to describe ablative surfaces [7, 10]. They are widely used in the hypersonic community because of their simplicity; they only require the definition of the probability that a macroscopic reaction takes place: 


$$
\gamma_{i}^{\text {rea }}=\frac{\mathcal{N}_{i}^{\text {rea }}}{\mathcal{N}_{i}}
$$

where $\mathcal{N}_{\mathrm{i}}^{\text {rea }}$ is the number flux of species i subject to the reaction and $\mathcal{N}_{\mathrm{i}}=n_{\mathrm{i}} \sqrt{k_{\mathrm{b}} T_{\mathrm{s}} /\left(2 \pi m_{\mathrm{i}}\right)}$, the number flux of species i impinging the surface. Symbol $k_{\mathrm{b}}$ stands for Boltzmann's constant, $T_{\mathrm{S}}$ the surface temperature, and $m_{\mathrm{i}}$, the mass of species i. A fully catalytic behavior is obtained when the probability $\gamma$ approaches unity; whereas a non-catalytic behavior is reached when $\gamma$ is equal to zero. The chemical production rate reads:

$$
\dot{\omega}_{\mathrm{i}}^{\mathrm{s}}=m_{\mathrm{i}} \gamma_{\mathrm{i}}^{\mathrm{rea}} \mathcal{N}_{\mathrm{i}}
$$

A previous numerical-experimental work [7] was performed to assess the nitridation efficiency on carbon surface:

$$
\gamma_{\mathrm{N}}^{\mathrm{CN}}\left(T_{\mathrm{S}}\right)=7.91 \cdot 10^{-2} \exp \frac{-5653}{T_{\mathrm{S}}}
$$

The major drawback of this phenomenological approach is the absence of pressure dependence, limiting its ability to describe the phenomenon at pressures different than the pressure used in the experiment (1500Pa).

\subsection{Finite-rate chemistry approach}

Alternative formulations to phenomenological approaches are Finite-Rate Chemistry (FRC) models describing each elementary reaction occurring on the surface (adsorption/desorption, nitridation, recombi

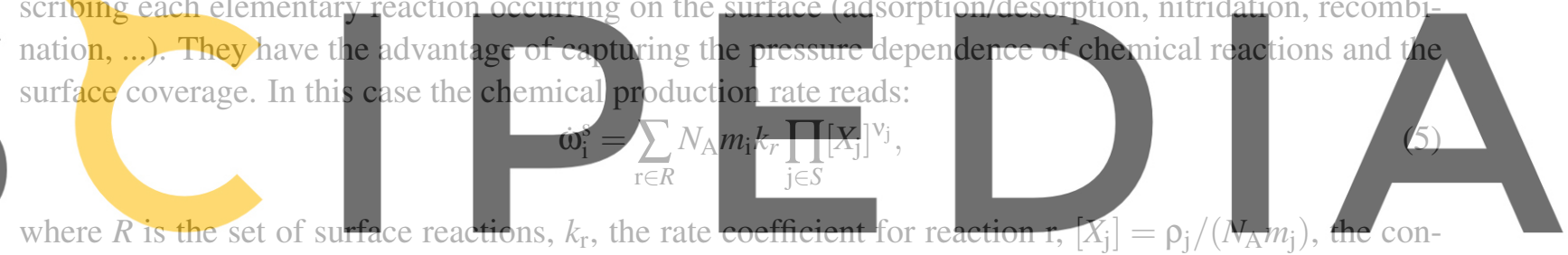
centration of species $\mathrm{j}, v_{\mathrm{j}}$, its stoichiometric coefficient, and $N_{\mathrm{A}}$, Avogadro's number. The FRC model

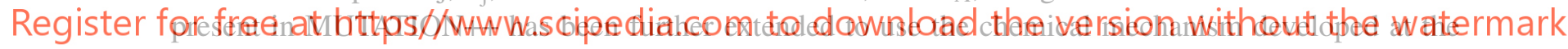
University of Minnesota for nitridation and nitrogen recombination [5]: the reactions considered are listed in table 1. The model was built from molecular beam experiment data obtained in low pressure conditions [4] and from Plasmatron data at a pressure of $1500 \mathrm{~Pa}$ [7]. According to the beam experiments, the probability of recombination of $\mathrm{N}$ atoms into $\mathrm{N}_{2}$ is about 10 times the one of $\mathrm{CN}$ formation. Such trend has its effects on the surface heat flux and the surface ablation, as it will be discussed in section 5.1. The rate coefficients for each surface reactions are also shown in table 1, where $B$ is the active site density on the surface $\left[\mathrm{mol} \mathrm{m}^{-2}\right], s$, the surface density of empty sites $\left[\mathrm{mol} \mathrm{m}{ }^{-2}\right], F_{\mathrm{N}}=\sqrt{k_{b} T / 2 \pi m_{\mathrm{N}}}$, a quarter of the mean thermal speed of atomic nitrogen, $F_{\mathrm{N}, 2 \mathrm{D}}=\sqrt{\pi k_{b} T / 2 m_{\mathrm{N}}}$, the mean thermal speed of the mobile adsorbed nitrogen on the surface, and $h$, the Planck constant. The set of equations (5) is closed by steady-state assumption $(d s / d t=0$, meaning that the net production of empty sites in time is zero) and the system is solved by the Newton method.

In order to compare our results with the ones reported in [7], an equivalent probability value was computed by dividing the chemical production rate term by the flux of the species impinging the surface:

$$
\gamma_{\mathrm{N}}^{\mathrm{CN}}=\frac{\dot{\omega}_{\mathrm{CN}}}{m_{\mathrm{CN}} \mathcal{N}_{\mathrm{N}}}, \quad \gamma_{\mathrm{N}}^{\mathrm{N} 2}=\frac{\dot{\omega}_{\mathrm{N} 2}}{m_{\mathrm{N}} \mathcal{N}_{\mathrm{N}}} \text {. }
$$


Table 1: FRC reactions for nitrogen flows[5].

\begin{tabular}{ccc}
\hline Reaction & Reaction Rate & Rate Coefficient \\
\hline $\mathrm{N}+\mathrm{s} \longrightarrow \mathrm{N}_{\mathrm{s}}$ & $\mathrm{k}_{1}[\mathrm{~N}][\mathrm{s}]$ & $\frac{F_{\mathrm{N}}}{B} \exp \frac{-2500}{T_{\mathrm{s}}}$ \\
$\mathrm{N}_{\mathrm{s}} \longrightarrow \mathrm{N}+\mathrm{s}$ & $\mathrm{k}_{2}\left[\mathrm{~N}_{\mathrm{s}}\right]$ & $\frac{2 \pi m_{\mathrm{N}} k_{b}^{2} T_{\mathrm{s}}^{2}}{N_{\mathrm{A}} B h^{3}} \exp \frac{-73971}{T_{\mathrm{s}}}$ \\
$\mathrm{N}+\mathrm{N}_{\mathrm{s}}+\mathrm{C}_{\mathrm{b}} \longrightarrow \mathrm{CN}+\mathrm{N}+\mathrm{s}$ & $\mathrm{k}_{3}[\mathrm{~N}]\left[\mathrm{N}_{\mathrm{s}}\right]$ & $\frac{F_{\mathrm{N}}}{B} 1.5 \exp \frac{-7000}{T_{\mathrm{s}}}$ \\
$\mathrm{N}+\mathrm{N}_{\mathrm{s}} \longrightarrow \mathrm{N}_{2}+\mathrm{s}$ & $\mathrm{k}_{4}[\mathrm{~N}]\left[\mathrm{N}_{\mathrm{s}}\right]$ & $\frac{F_{\mathrm{N}}}{B} 0.5 \exp \frac{-2000}{T_{\mathrm{s}}}$ \\
$\mathrm{N}_{\mathrm{s}}+\mathrm{N}_{\mathrm{s}} \longrightarrow \mathrm{N}_{2}+2 \mathrm{~s}$ & $\mathrm{k}_{5}\left[\mathrm{~N}_{\mathrm{s}}\right]\left[\mathrm{N}_{\mathrm{s}}\right]$ & $\sqrt{\frac{N_{\mathrm{A}}}{B} F_{\mathrm{N}, 2 \mathrm{D}} 0.1 \exp \frac{-21000}{T_{\mathrm{s}}}}$ \\
$\mathrm{N}_{\mathrm{s}}+\mathrm{C}_{\mathrm{b}} \longrightarrow \mathrm{CN}+\mathrm{s}$ & $\mathrm{k}_{6}\left[\mathrm{~N}_{\mathrm{s}}\right]$ & $10^{8} \exp \frac{-20676}{T_{\mathrm{s}}}$ \\
\hline
\end{tabular}

\section{GOVERNING EQUATIONS AND FLOW SOLVERS}

The gas is a mixture of $n_{s}$ chemical reacting species in thermal equilibrium. The flow is described by the Navier-Stokes equations:

$$
\begin{aligned}
& \frac{\partial \rho_{\mathrm{i}}}{\partial t}+\nabla \cdot\left(\rho_{\mathrm{i}} \mathbf{u}+\dot{j}_{\mathrm{i}}\right)=\dot{\omega}_{\mathrm{i}}, \quad \forall \mathrm{i} \in S, \\
& \frac{\partial \rho \mathbf{u}}{\partial t}+\nabla \cdot(\rho \mathbf{u} \cdot \mathbf{u}+p \overline{\overline{\mathbf{I}}}+\overline{\bar{\tau}})=0, \\
& \frac{\partial \rho E}{\partial t}+\nabla \cdot(\rho \mathbf{u} H+\overline{\bar{\tau}} \mathbf{u}+\mathbf{q})=0 .
\end{aligned}
$$

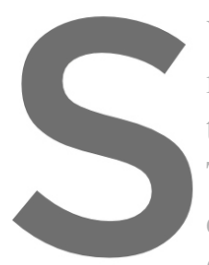

Vector $\mathbf{u}$ is the mass-aver modynamic pressure of th total enthalpy, and $\mathbf{q}$, the Thermodynamics properties Chapman-Enskog solution
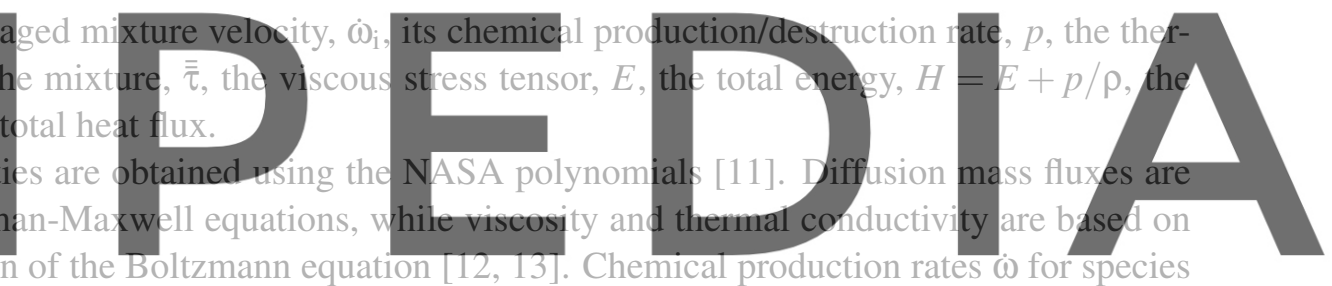

$i$ are computed using finite-rate chemistry, with reaction rate coefficients taken from [14]. The library

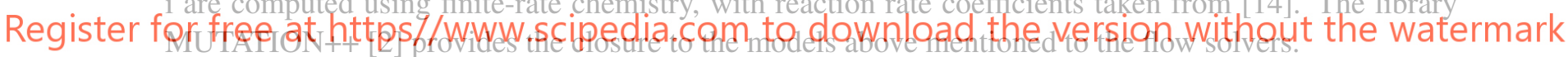

\subsection{VKI stagnation line solver}

When dealing with axisymmetric problems, a Dimensionally Reduced Navier-Stokes Equations (DRNSE) formulation [15] can be used to simulate the flowfield on the stagnation line. The system is written in spherical coordinates $(r, \theta, \phi)$, then, exploiting the axial symmetry, $u_{\phi}=\partial / \partial \phi=0$ and letting $\theta \rightarrow 0$, the DRNSE formulation is obtained. Munafò [6] developed a software for the DRNSE equations based on a finite volume discretization method, together with an implicit local time-stepping technique to reach the steady state. The code interfaced to MUTATION++ helps us to verify the coupling of US3D to MUTATION++, using the Roe scheme [16] for the numerical fluxes.

\subsection{US3D solver}

US3D is a three-dimensional finite-volume flow solver developed at the University of Minnesota for aerodynamic/hypersonic applications [8]. The code uses an unstructured grid, the numerical fluxes are computed in this work according to the modified Steger-Warming scheme [18] with a MUSCL approach 
to obtain second-order accuracy. The Data Parallel Line Relaxation (DPLR [17]) ensures rapid convergence to steady-state. US3D is highly scalable and efficient, making it possible to solve very large problems on parallel computers in a cost-effective manner. We have coupled US3D to MUTATION++ by means of User defined routines available in the code for the closure of: I) Transport (viscosity, thermal conductivity, diffusive fluxes), II) Gas chemistry, III) Multi-temperature energy transfer terms, and IV) Gas-Surface interaction.

\section{NITRIDATION EXPERIMENTAL CAMPAIGN}

Numerical simulations were performed to reproduce the data obtained in an experimental campaign carried out in the VKI Plasmatron facility to study low pressure nitridation (1500 Pa) [7]. It consists of a nitrogen plasma flow over a $25 \mathrm{~mm}$ radius hemisphere carbonaceous sample. The surface temperature was measured by means of a calorimeter, the recession rate by using a high-speed camera and the temperature in the BL with a spectrometer. Free stream conditions were numerically rebuilt; further information is provided in [7]. Input conditions for the simulations, as well as surface measurements are reported in table 2. The chemical composition at the inlet of the simulation was considered to be in equilibrium. The

Table 2: Overview of Plasmatron test conditions for a nitrogen flow at $1500 \mathrm{~Pa}$ over a $25 \mathrm{~mm}$ radius hemisphere carbonaceous sample [7]: dynamic pressure $\mathrm{P}_{\mathrm{d}}$, generator power $\mathrm{P}_{\mathrm{el}}$, sample mean temperature $\mathrm{T}_{\mathrm{s}}$, mass blowing rate $\dot{m}$, nitridation efficiency $\gamma_{\mathrm{N}}^{\mathrm{CN}}$, boundary layer edge conditions (density $\rho_{\text {edge, }}$, temperature $\mathrm{T}_{\text {edge, }}$, and velocity $\mathrm{u}_{\text {edge }}$ ).
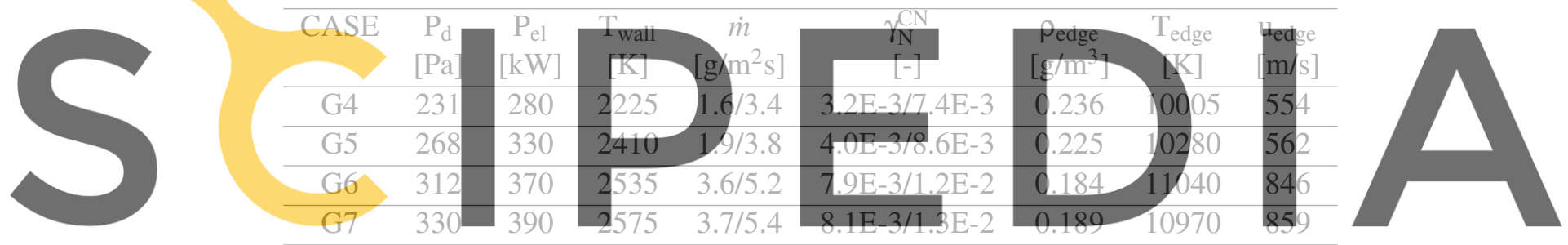

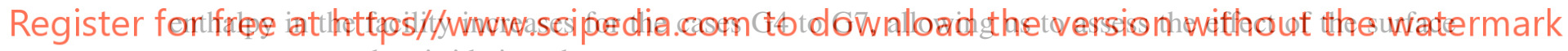
temperature on the nitridation phenomenon.

\section{SIMULATIONS RESULTS}

Both the VKI stagnation line and US3D flow solvers were used to simulate the Plasmatron experimental campaign. Solving the DRNSE equations can be practical for axisymmetric problems given the reduced dimension of the problem. In sec. 5.1, we validate the FRC model by comparing the simulation results obtained by means of the VKI stagnation line flow solver coupled to MUTATION++. In sec. 5.2, we verify the implementation of the coupling of US3D to MUTATION++ based on a code-to-code comparison with the stagnation line flow solver.

\subsection{Stagnation line solver results}

A 1-D mesh of 730 points was generated based on a hyper-tangent function with a stretching factor equal to five. Simulations were carried out for the conditions mentioned in table 2 for a mixture composed of the following species: $\mathrm{N}, \mathrm{N}_{2}, \mathrm{~N}^{+}, \mathrm{e}^{-}, \mathrm{CN}, \mathrm{C}, \mathrm{C}^{+}, \mathrm{C}_{2}, \mathrm{C}_{3}$. Surface results based on the FRC models were post-processed by means of equation (6) to compute the values of the nitridation and nitrogen 
recombination efficiencies. Results for the four experimental cases given in table 2 are shown in fig. 2 together with the predicted mass blowing rate and the related uncertainties reported in [7].

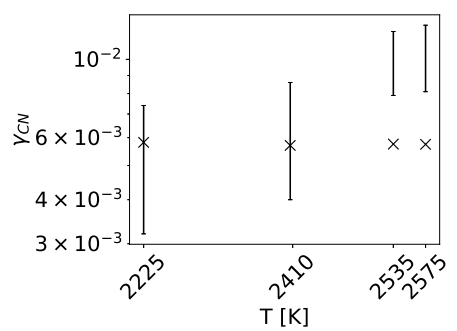

(a) Nitridation efficiency.

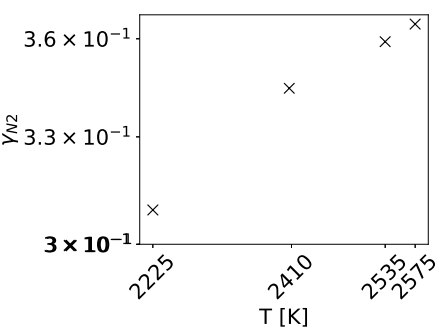

(b) Nitrogen recombination efficiency.

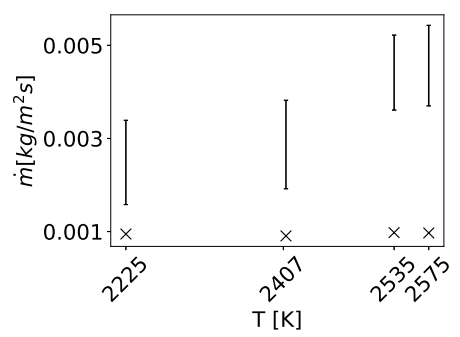

(c) Mass blowing rate.

Figure 2: Solution obtained with the stagnation line code relative to a nitrogen flow over a $25 \mathrm{~mm}$ radius hemisphere carbonaceous sample: quantities of interest at the stagnation point for the four cases detailed in table 2 are plotted as function of the surface temperature (cross). Nitridation efficiency and mass blowing rate from [7] are reported with error bars.

The value of the nitridation probability $\gamma_{\mathrm{CN}}$ given in fig. $2 \mathrm{a}$ is within the uncertainty reported for the first two cases, while it is underpredicted for the second two cases. The recombination at the wall is more important for higher temperatures, as shown in fig. $2 b$. The recombination efficiency is not reported

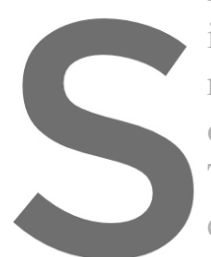
in [7]. Recombination reaction reaction (fig. 5c) and, thus, on $\mathrm{CN}$ production, is underprediatel Though the FRC model experiments, the model takes into in [7]. Inclusion of both nitridation and recombination reactions allows for accurate model calibration through direct flow simulations șuch as those performed in the current work, rather, than relying on OD

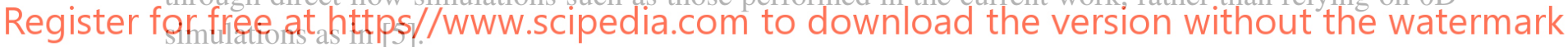

\subsection{US3D axisymmetric simulation}

This section focuses on the code-to-code verification strategy by simulating the same experimental conditions using US3D. Results were extracted on the stagnation line and compared to the ones obtained from the VKI stagnation line flow solver.

The axisymmetric computational domain is represented in fig. 3. It consists of a uniform inlet, whose state (density, temperature, and velocity) is reported in table 2 assuming local thermodynamic equilibrium. The chamber pressure $(1500 \mathrm{~Pa})$ was prescribed at the outlet. On the sample, the surface mass balance eq. (1) was imposed, together with the FRC closure. Past the hemispherical part of the sample (at $x=0.0 \mathrm{~m}$ ), an isothermal, non reacting surface boundary condition was imposed. The grid independence was assessed by doubling the number of nodes in the $\mathrm{x}$ and $\mathrm{y}$ directions (table 3 ) and verifying that the surface properties (pressure, heat flux, mass blow rate) remain unchanged, as shown in fig. 4.

Results were extracted from the solution along the stagnation line and compared to the ones returned by the VKI solver. As we can see in fig. 5, the agreement between the two codes is excellent for all the 


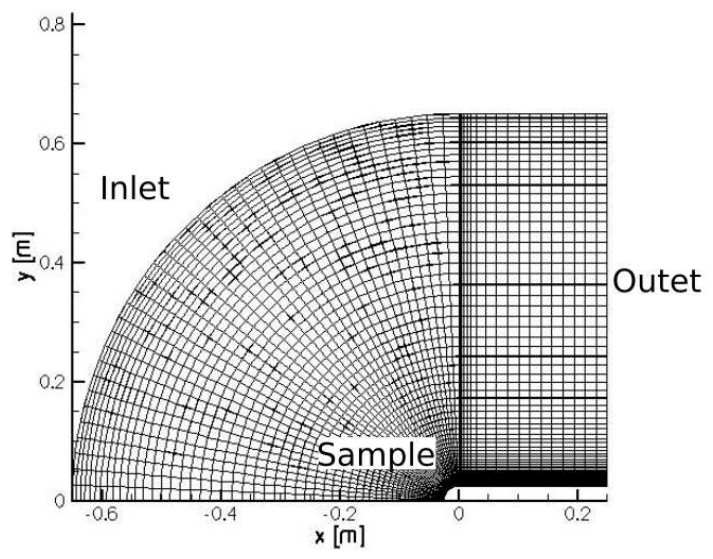

Figure 3: Numerical grid used for US3D simulations to solve the axisymmetric flowfield of the nitridation experimental camnaign (sec. 4). Mesh 1 of table 3.

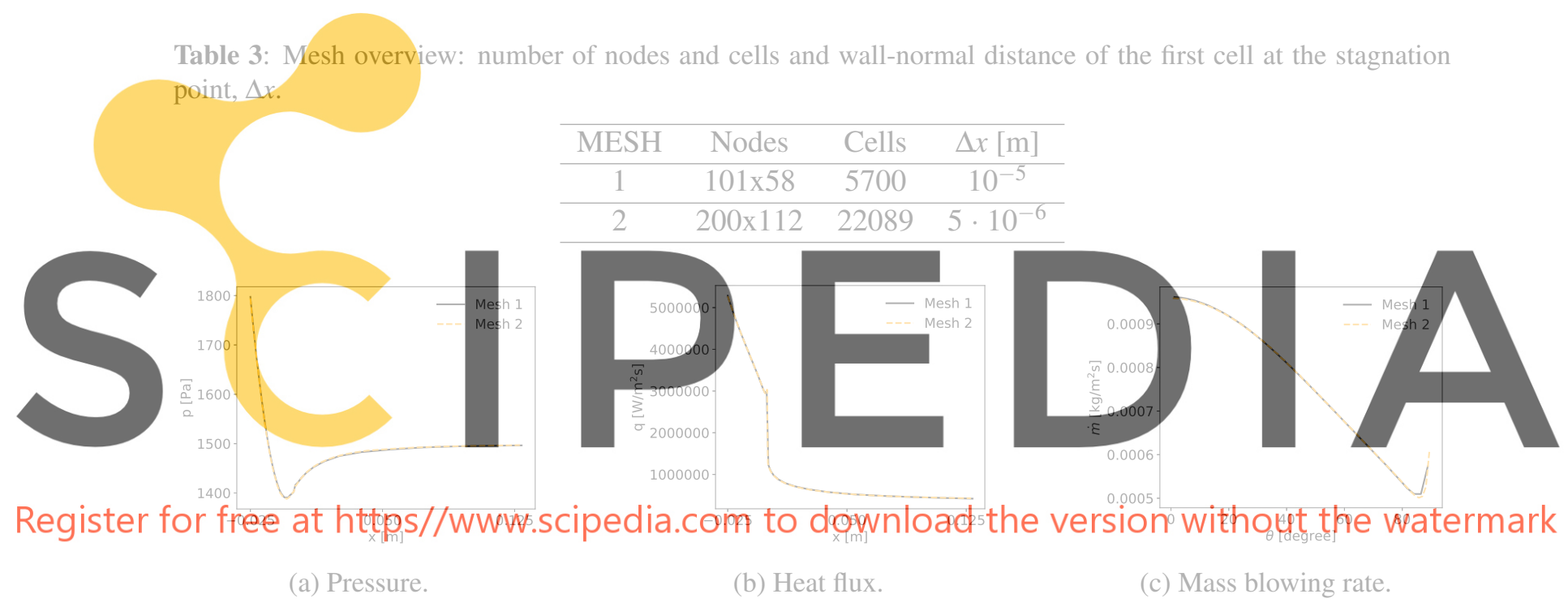

Figure 4: Comparison of the numerical results on the surface obtained using US3D between the two different meshes given in table 3 for the test-case G5 of table 2. Pressure and heat flux are plotted as a function of the $\mathrm{x}$-coordinate, and the mass blowing rate as a function of the angular coordinate.

quantities of interest (temperature, velocity and mass fraction distribution). The small differences in the results can be attributed to the different sets of equations solved and the numerical methods. In fig. 6a, the angular distribution of the mass blowing rate is shown for the four test cases. Also in this case, the differences between the results obtained by the two solvers are minimal, verifying the implementation of the boundary condition. An interesting feature that can be quantified from the US3D solution is the increase in the mass blowing rate at the end of the hemispherical sample. This can be explained by looking at fig. $7 \mathrm{a}$, where it appears clear that the mass blowing rate inverts the trend after the recompression point: this increases the flux of atomic nitrogen impinging the sample and thus its surface chemical activity. 


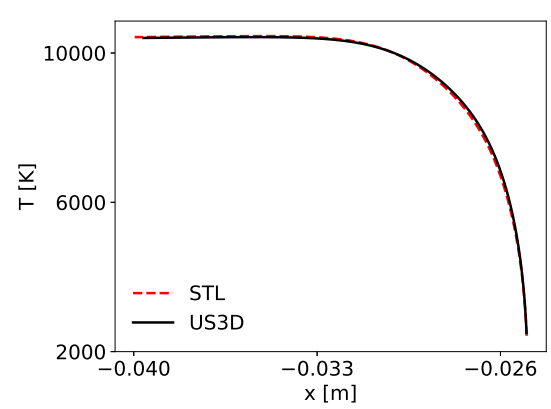

(a) Temperature.

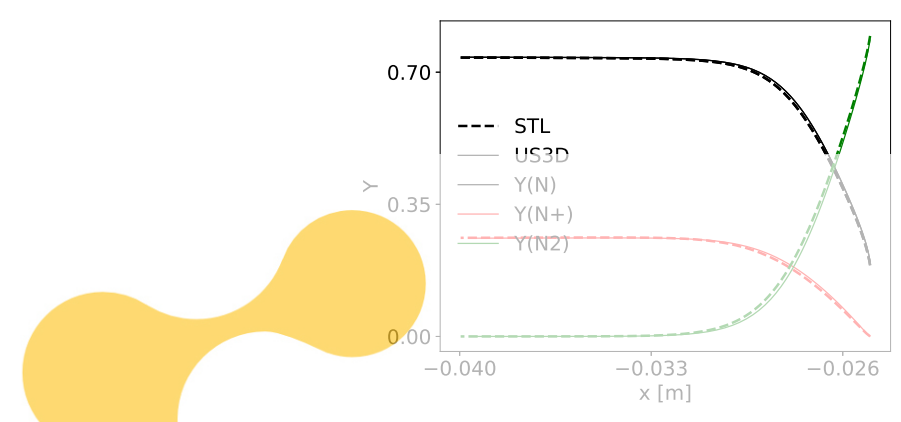

(c) Mass fractions.

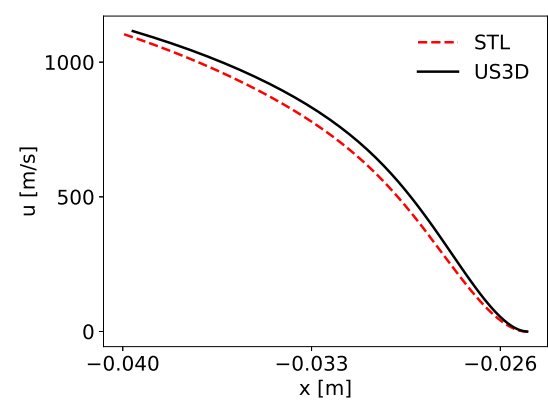

(b) Velocity.

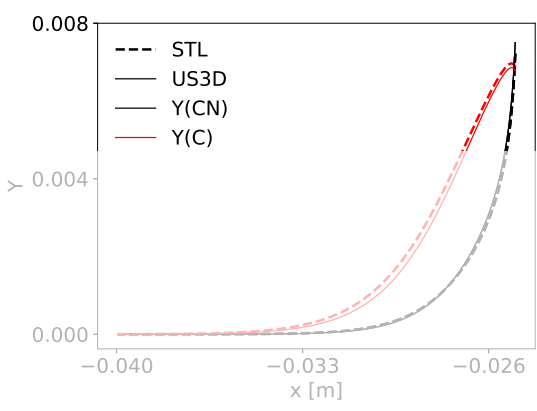

(d) Mass fractions.

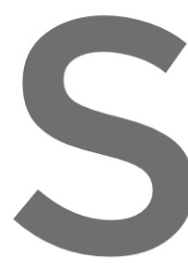

Figure 5: Simulation results

in the US3D simulation within the code (STL in the legend)
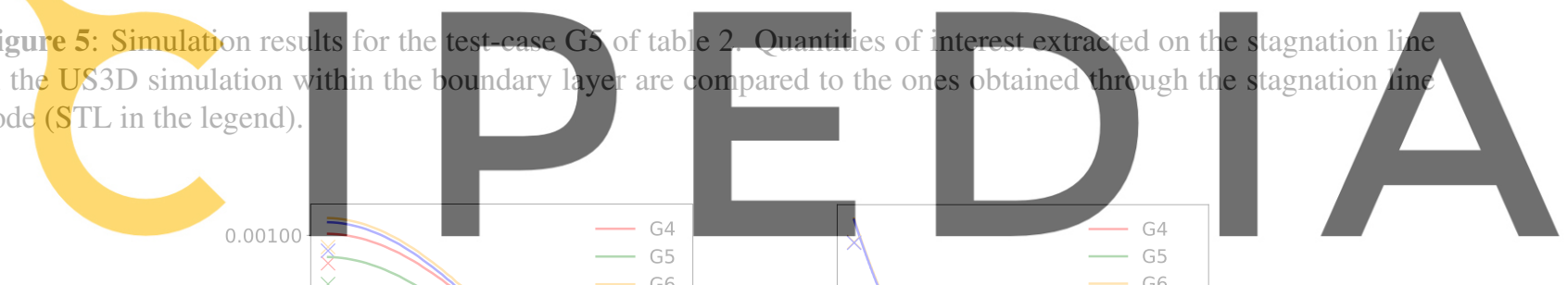

Register for free at htEps//www.scipedia.com $€ \succeq$ do

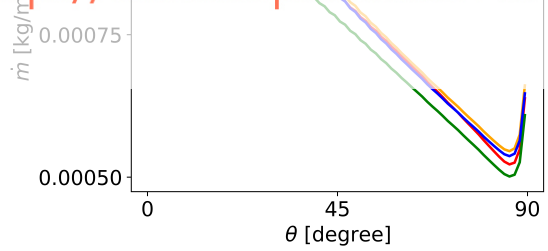

(a) Mass blowing rate.

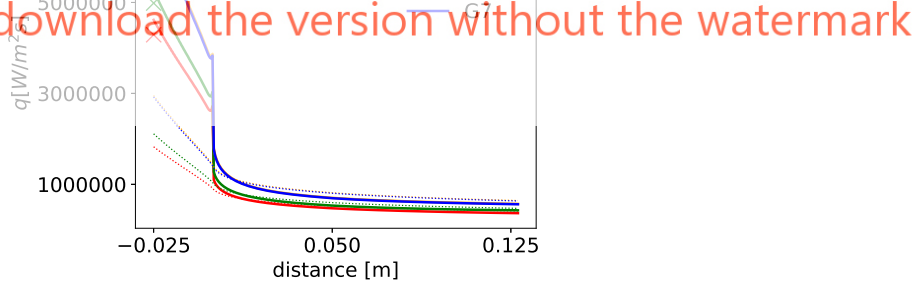

(b) Heat Flux.

Figure 6: Quantities of interest for the four cases detailed in table 2. US3D surface results (lines) are plotted against the stagnation point values from the stagnation line code (cross). Mass blowing rate is plotted as function of angular coordinate, and the heat flux as function of $\mathrm{x}$-coordinate. The heat flux plot contains the results for a reactive surface (solid lines), and also for an inert surface (dotted lines).

Another important quantity is the heat flux distribution along the sample, plotted in fig. $6 \mathrm{~b}$. The value obtained using the FRC model (continuous line) is shown against the one predicted by assuming an inert surface (dotted line). Specifically, in the hemispherical part, we can see that the strong catalytic nature 
of the surface in the FRC model, increases the surface heat flux by 3 times.

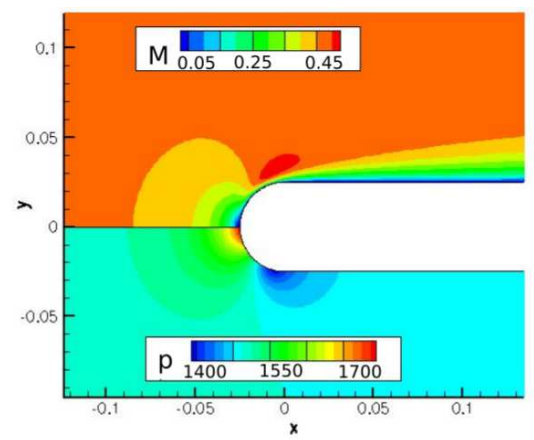

(a) Pressure and Mach number.

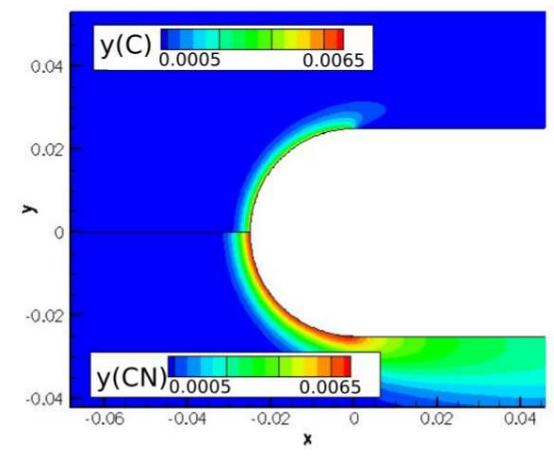

(b) $\mathrm{C}$ and $\mathrm{CN}$ mass fraction.
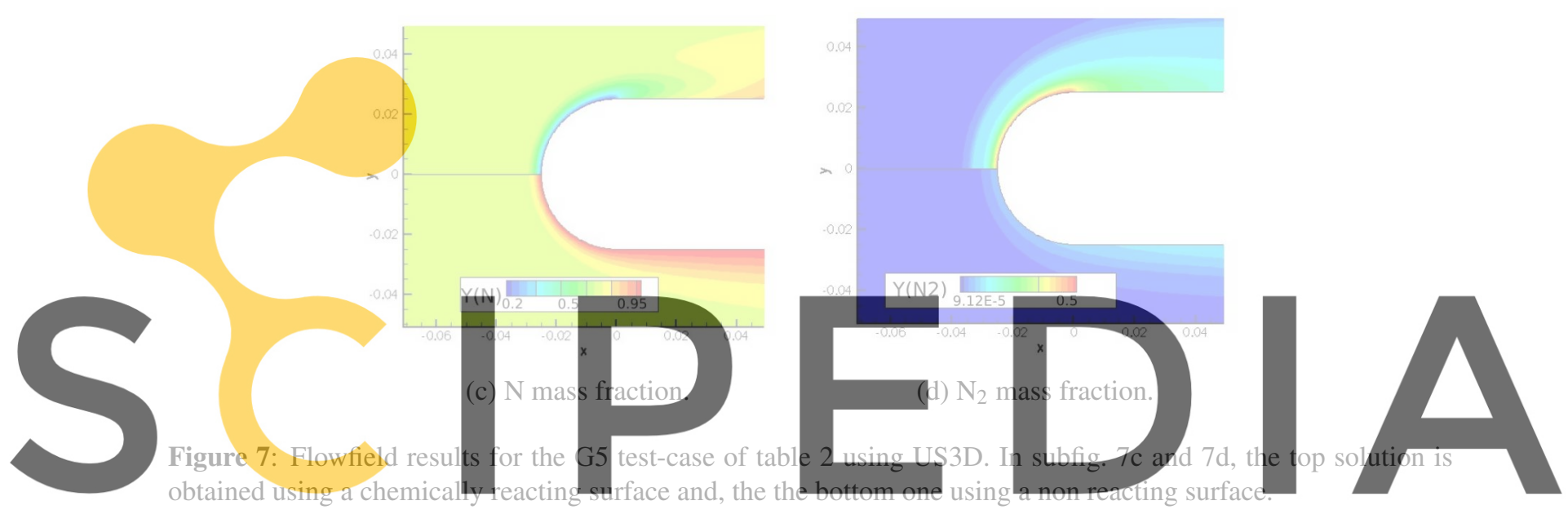

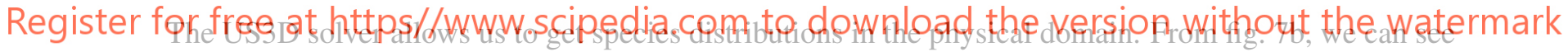

that $\mathrm{CN}$ produced at the wall dissociates into $\mathrm{N}$ and $\mathrm{C}$ via gas phase chemical reactions, and then, that

the carbon diffuses out from the wall. Furthermore, in fig. $7 \mathrm{c}$ and $7 \mathrm{~d}$ we can see the distribution of the atomic and molecular nitrogen in the case of a chemically reacting and a non-reacting surface. Around a chemically reactive surface (shown on the top half of each figure), the boundary layer is dominated by molecular nitrogen due to the recombination reaction at the surface. This is in contrast with the results obtained around a chemically inert (non-reactive) surface shown on the bottom half of each picture, which is characterized by the presence of atomic nitrogen.

\section{CONCLUSIONS AND FUTURE WORK}

The FRC model for treating nitrogen recombination and nitridation of $\mathrm{N}$ atoms on a carbon surface was plugged into the open source library MUTATION++. A nitridation campaign conducted at the von Karman Institute was simulated using both the VKI stagnation line code and the US3D solver for verification and validation purposes.

It was shown that the FRC model underpredicts the mass blowing rate reported experimentally and exhibits an important catalytic behaviour, in agreement with beam experiments. As a result, the surface 
experiences a strong heat flux, while decreasing the number of nitrogen atoms available for nitridation. Recombination must be taken into account for correctly predicting the surface response. The coupling of the FRC model with a flow solver allows for further model calibration by comparing simulation results with Plasmatron data.

The coupling between the gas-surface interaction module implemented in MUTATION++ and US3D allows us to perform high-fidelity simulations of complex flows/geometry in hypersonic applications. This coupling was verified through a code-to-code strategy: results were extracted along the stagnation line and compared to the ones obtained by means of the VKI stagnation line code. The comparison showed excellent agreement, verifying the implementation.

\section{ACKNOWLEDGMENTS}

Authors acknowledge the Air Force Office of Scientific Research (AFOSR) for supporting the work and for sponsoring the collaboration between the von Karman Institute and the University of Minnesota under the grant FA9550-18-1-0209.

\section{REFERENCES}

[1] Bellas-Chatzigeorgis, G. and Turchi, A. and Viladegut, A. and Chazot, O. and Barbante, P.F, and Magin, T. Development of catalytic and ablative gas-surface interaction models for the simulation of reacting gas mixtures. 23rd AIAA Computational Fluid Dynamics Conference (2017) $10.2514 / 6.2017-4499$.

[2] Scoggins, J. B. and Leroy, V. Bellas-Chatzigeorgis, G. and Dias, B. Magin, T. E. Mutation: MUlticomponent Thermodynamic And Transport properties for IONized gases in C++. SoftwareX (2020) 12:100575.

[3] Bellas-Chatzigeorgis, G. and Barbante P.F and Magin, T. E. Energy Accommodation Coefficient Calculation Methodology Using State-to-State Catalysis Applied to Hypersonic Flows. AIAA Journal (2020) 58:1,278-290.

[4] Murray,V. J. and Recio, P. and Caracciolo, A. and Miossec, C. and Balucani, N. and Casavecchia, P. and Minton, T. K. Oxidation and nitridation of vitreous carbon at high temperatures. Carbon (2020) 167:388-402.

[5] Prata, K. S. and Minton, T. and Schwartzentruber, T. E. Air-Carbon Ablation Model for Hypersonic Flight from Molecular Beam Data. ChemRxiv. Preprint (2020) 10.26434/chemrxiv.12959972.

[6] Munafò, A., Magin, T. Modeling of stagnation-line nonequilibrium flows by means of quantum based collisional models. Physics of Fluids (2014) 26:9,097102.

[7] Helber, B., Turchi, A., Magin, T. Determination of active nitridation reaction efficiency of graphite in inductively coupled plasma flows. Carbon (2017) 125:582-594.

[8] Candler, G., V. and Heath, J. and Nompelis, I. Gidzak, V. and Subbareddy, P. and Barnhardt, M. Development of the US3D Code for Advanced Compressible and Reacting Flow Simulations. 53rd AIAA Aerospace Sciences Meeting (2015) 10.2514/6.2015-1893.

[9] Goulard, R. On Catalytic Recombination Rates in Hypersonic Stagnation Heat Transfer. Journal of 
Jet Propulsion (1958) 28:11,737-745.

[10] Park, C. Effects of Atomic Oxygen on Graphite Ablation. AIAA journal (1976) 14:11,1640-1642.

[11] Gordon, S. and McBride, J. Thermodynamic Data to $20000 \mathrm{~K}$ for Monatomic Gases. Tech. Rep. TP 208523, NASA (1999).

[12] Ferziger, J. H. and Kaper, H. G. Mathematical Theory of Transport Processes in Gases. American Journal of Physics (1973) 41:4,601-603.

[13] Magin, T. E. ,and Degrez, G. Transport algorithms for partially ionized and unmagnetized plasmas. Journal of Computational Physics (2004) 198:2,424-449.

[14] Olynick, D. and Chen, Y. and Tauber, M. Aerothermodynamics of the Stardust Sample Return Capsule. Journal of Spacecraft and Rockets (1999) 36:3,442-462.

[15] Klomfass, A. and Fur Mechanik, L. F. and Müller, S. A Quasi-Onedimensional Approach for Hypersonic Stagnation-Point Flows. Technical report, Internal Report (1965).

[16] Roe, P. Approximate Riemann solvers, parameter vectors, and difference schemes. Journal of Computational Physics (1981) 43:2,357-372.

[17] Wright, M.J. and Bose, D. and Candler, G.V. A Data-Parallel Line Relaxation Method for the Navier-Stokes Equations. 53rd AIAA Journal (1998) 36:9,1603-1609.

[18] Steger, J. and Warming, R.W. Flux Vector Splitting of the Inviscid Gasdynamics Equations with Application to Finite Difference Methods. Journal of Computational Physics (1981) 40:2,263-293. 